\title{
Electro-optic beam manipulation through photorefractive needles
}

\author{
Eugenio DelRe, Bruno Crosignani, and Paolo Di Porto \\ Dipartimento di Fisica, Università dell'Aquila, 67010 Coppito-l'Aquila, Italy, and Istituto Nazionale Fisica della Materia, \\ Unità di Roma "La Sapienza," 00185 Rome, Italy \\ Elia Palange \\ Dipartimento di Energetica and Istituto Nazionale Fisica della Materia, Università dell'Aquila, 67040 Monteluco di Roio-l'Aquila, Italy \\ Aharon J. Agranat \\ Department of Applied Physics, Hebrew University of Jerusalem, Jerusalem 91904, Israel \\ Received July 26, 2002

\begin{abstract}
We demonstrate electro-optic spatial two-dimensional mode switching in a bulk sample of potassium lithium tantalate niobate. Spatial confinement, mode coupling, and electro-optic functionality are mediated by two photorefractive needle solitons of opposite electroholographic charges embedded together in their anisotropic lobular structure. (C) 2002 Optical Society of America

OCIS codes: $190.5330,230.2090$.
\end{abstract}

When speed, functionality, and miniaturization require transparent optical technology, all-optical functionality based on direct nonlinear electronic polarizability is generally abandoned in favor of the more restrictive linear electro-optic beam manipulation in which the electronic and optical signals have no feedback. More physically elaborate avenues are based on secondary processes that enhance the effective optical nonlinearity. Such is the case for photorefractive phenomena observed in lightly doped ferroelectrics. Here wave mixing and self-action offer a family of optical functions. Photorefractive solitons, ${ }^{1,2}$ which have opened the way to an array of novel nonlinear effects, are considered one of the promising avenues for achieving a new generation of beam-steering and -manipulation devices. ${ }^{3}$ The observation of twodimensional solitons, ${ }^{4,5}$ i.e., beams that propagate in a bulk environment without suffering diffraction or distortion in both transverse directions (needle solitons), permits the integration of a bulk material with the confined propagation of an optical circuit. Circumventing the refined and costly development of a waveguide growth technology, needle self-trapping opens the door to guided coupling from one spatial mode to another in three dimensions.

Photorefractive needles are supported by the spatially modulated electro-optic response to the photogenerated charge that is associated with weak absorption at visible wavelengths. They can guide information-bearing infrared streams through a sample in a linear and transparent manner. Pioneering devices have been demonstrated that range from simple signal guiding ${ }^{6}$ to more-complex systems such as directional couplers, ${ }^{7}$ solitoninteraction-based beam dividers, ${ }^{8}$ and secondharmonic-parametric-oscillator enhancers. ${ }^{9,10}$

Devices are hampered by the slow time scales of the underlying charge dynamics, characterized by multistage hopping through the dielectric before equilibrium distribution is achieved. ${ }^{3}$

0146-9592/02/242188-03\$15.00/0
A different situation is found for solitons that occur in centrosymmetric crystals, such as potassium lithium tantalate niobate, for which quadratic, as opposed to linear, electro-optic modulation makes electroholography possible. ${ }^{11}$ Solitons are a result of the redistribution of charge that leads to a space-charge field $\mathbf{E}_{\mathrm{sc}}(\mathbf{r})$ in response to a given value of external bias field $E=E_{\text {sol }}$. Once all slow-charge dynamics have ceased, the application of different external bias fields $E$ to the sample gives rise to different bulk index patterns $\Delta n(\mathbf{r})$. This is a direct consequence of the nonlinear combination of the photoinduced space-charge field $\mathbf{E}_{\mathrm{sc}}(\mathbf{r})$ and $E$ and is not limited by any intermediate time scale; it is a purely electro-optic modulation. Studies of one-dimensional slab solitons show that electro-optic functionality can be exploited to couple, route, and modulate light. ${ }^{12}$ An obstacle to direct extension of this functionality to needles is the distorted and highly elliptical nature of the underlying anisotropic lobular patterns. ${ }^{13}$

In this Letter we describe experiments that led to what is to our knowledge the first demonstration of practical electro-optic beam manipulation based on the electro-optic functionality associated with a pair of photorefractive needles of opposite electroholographic charge. In a first scheme we locked the two needles together by embedding one in the anisotropic lobular structure of the other. In a second, more-involved scheme, we partially circumvented anisotropy by stacking the two needles in the direction orthogonal to the external bias. This system permits efficient and intrinsically fast electro-optic spatial mode switching in a bulk crystal sample, self-integrated into a fiber-based circuit through the formation of spatial solitons.

The experimental device is similar to previous ones. ${ }^{3,5}$ A continuous-wave visible $\lambda=514 \mathrm{~nm} \mathrm{Ar}^{+}$ laser beam is first expanded and then focused to a micrometer-sized waist onto the input facet of a zero-cut sample of $3.7^{(x)} \mathrm{mm} \times 4.1^{(y)} \mathrm{mm} \times 2.4^{(z)} \mathrm{mm}$ 
potassium lithium tantalate niobate. The beam, launched in the $z$ direction, is linearly polarized along the $x$ axis, which is also the direction of external biasing field $E$. The crystal is mounted upon a micrometric transverse $(x, y)$ translation stage. A feedback Peltier junction fixes the sample temperature to $T \simeq 24^{\circ} \mathrm{C}$. The sample manifests a second-order phase transition from its centrosymmetric phase to the polar ferroelectric structure at $T_{c} \simeq 11^{\circ}$ and is transparent, apart from a small concentration of copper and vanadium impurities that give rise to considerable photorefraction for $\lambda<600 \mathrm{~nm}$. Beam dynamics are observed by imaging of the $(x, y)$ planes onto a beam analysis apparatus. No background illumination is implemented, and all phenomenology reported is therefore associated with the plateau region of quasi-steady-state self-trapping, ${ }^{3}$ which permits multistage circuit imprinting. We demonstrate electro-optic functionality by applying different values of $V$ to the sample (i.e., $E=V / L$ ); then the crystal is appropriately positioned with respect to the information-carrying read beam. This signal, which can be at a fully transparent $(\lambda>600 \mathrm{~nm})$ wavelength (1.3-1.6 $\mu \mathrm{m}$ for telecommunication devices), is here the same soliton-generating beam, strongly attenuated and appropriately displaced to fruitfully mimic signal behavior. ${ }^{12}$ In our experiments the $0.7-\mu \mathrm{W}$ write beam gives rise to nonlinear dynamics with a time scale $\tau_{\text {write }} \simeq 30 \mathrm{~s}$, whereas the 35-nW signal-read beam propagates linearly, because its dynamic time scales $\tau_{\text {signal }} \simeq 20 \tau$ are much longer (dynamics would be totally absent for $\lambda>600 \mathrm{~nm}$ ).

We have thus identified the most basic switching technique in the formation of a two-needle system of appropriate electroholographic charge, i.e., the polarity of the external field $E$ leading to soliton formation, which occurs for both $E_{\mathrm{sol}}$ and $-E_{\mathrm{sol}} .{ }^{5}$ It it imprinted in the soliton-supporting screening charge distribution. If a positive soliton is formed by biasing of the sample with $V=+V_{\text {sol }}$ and is supported by the resulting $\mathbf{E}_{\text {sc }}$, its negative optical twin, formed with $V=-V_{\text {sol }}$, is supported by $-\mathbf{E}_{\mathrm{sc}}$. Most importantly, in the readout phase the two behave in a diametrically opposite manner: For $V=+V_{\text {sol }}$ the positive needle will be guiding, but the negative needle one will be strongly antiguiding. Conversely, for $V=-V_{\text {sol }}$ the positive needle will become strongly antiguiding, whereas the negative needle will form a solitonsupporting guiding index pattern.

The possible two-needle configurations are numerous, depending on their positions and charges in relation to the intrinsic needle anisotropy and on their consequent mutual interaction. ${ }^{3}$ We can identify two major classes of needle pairs: equally charged and oppositely charged pairs. In the first case, to achieve electroholographic switching we must make use of the oppositely charged lobes along the $x$ axis. ${ }^{13,14}$ If the two solitons are generated, for example, in sequence (thus reducing direct dynamic interaction) and are positioned in such a way as to share one lobe exactly, the distortion encountered in single-soliton electroholography ${ }^{13}$ is partially compensated for by the more pronounced guiding pattern that is due to the su- perposition of the lobes of the two needles. This compensation, however, is strongly dependent on the relative position of the two needles.

A more fruitful approach to achieving electroholographic switching is based on oppositely charged pairs, in which the single guided modes stem from soliton-supporting patterns. For spatially distinct positions the opposite charge guarantees that the application of opposite polarities will produce two distinct alternatively guiding and antiguiding modes. Given the relevant intersoliton interaction, the most reproducible configuration is achieved as follows: First we generate a positive needle [Figs. 1(a)-1(c)]; then we embed a second, negative, needle centered onto a sidelobe of the first needle [Figs. 1(e) and 1(f)]. After the first 9- $\mu \mathrm{m}$ FWHM soliton has formed, for $V=V_{\text {sol }}=1.2 \mathrm{kV}$, we set $V=0$ and reposition the crystal to launch the beam onto the now guiding external lobe $(\Delta x \simeq 16 \mu \mathrm{m})$. Setting $V=-V_{\text {sol }}$, we form a second negative needle. This procedure permits the direct alignment of the pair without introducing an extraneous spatial scale, i.e., a shift between the solitons, and the almost total attenuation of the strong antiguiding associated with the positive soliton. This antiguiding pattern, which actually protects the first needle structure during the second phase of writing, would otherwise render the sequence impossible at such close proximity. The second (negative) soliton, which forms on the nonlocal tails of the positive soliton, has a $10-\mu \mathrm{m}$ FWHM. We now turn to the readout phase, appropriately lowering the launch intensity and repositioning the crystal to feed the positive soliton; no further mechanical alteration is introduced. We achieve electro-optic mode switching by commuting the applied voltage from $+V_{\text {sol }}$ to $V_{- \text {sol }}$, as in Figs. 2(a)-2(c). The $16-\mu \mathrm{m}$ beam shift and mode switching are a consequence both of the guiding pattern of the guiding needle and of the antiguiding pattern of the oppositely charged needle. In Fig. 2 we quantify switching by comparing the optical intensity
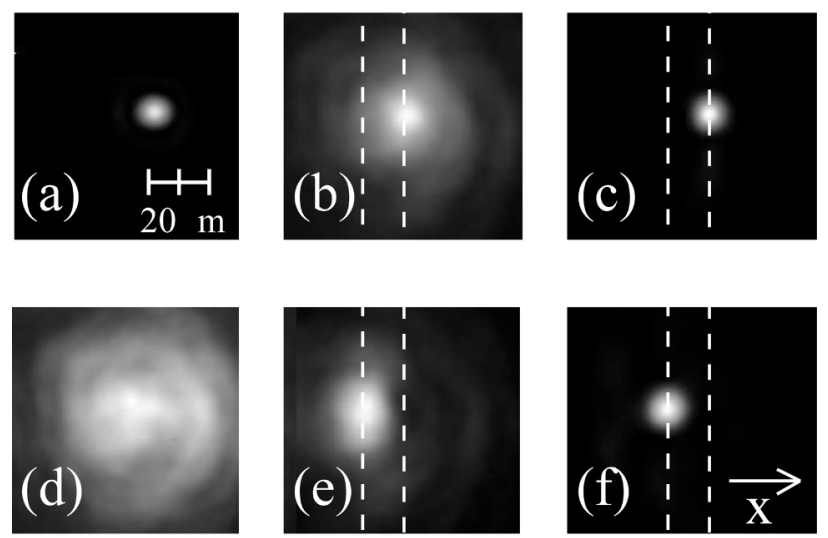

Fig. 1. Embedding a negatively charged needle in the lobular structure of a positive one. (a) Transverse intensity distribution of the input $9-\mu \mathrm{m} \mathrm{FWHM} \mathrm{TEM}_{00}$ beam, (b) linear output diffraction to $22 \mu \mathrm{m}$, (c) output needle formation for $V=1.2 \mathrm{kV}$, (d) output readout at $V=0$, (e) output when the beam is launched onto the lateral lobe at $V=0$ after a $16-\mu \mathrm{m}$ shift, (f) oppositely charged needle output for $V=-1.2 \mathrm{kV}$. 

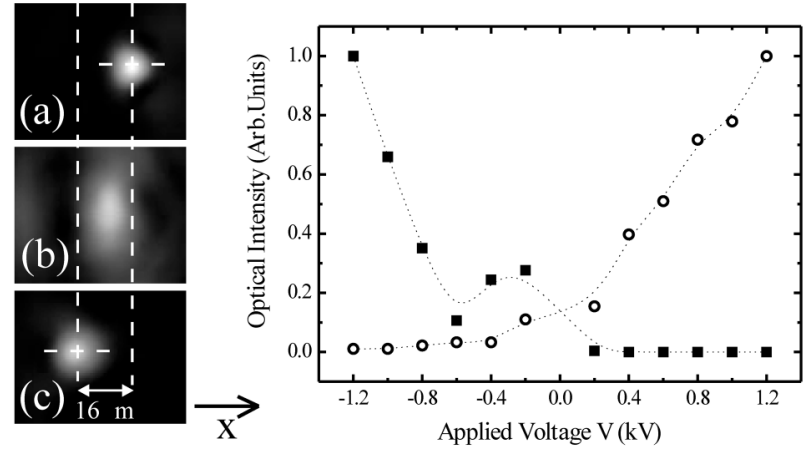

Fig. 2. Electro-optic mode switching. Output read intensity distribution for (a) $V=1.2 \mathrm{kV}$, (b) $V=0$, and (c) $V=-1.2 \mathrm{kV}$. Right, comparison of optical intensity at the two output mode peaks as a function of $V$; circles (squares), positive (negative) mode.

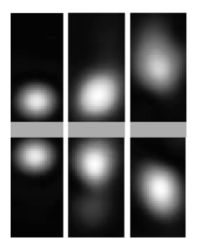

(a) (b) (c)

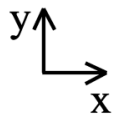

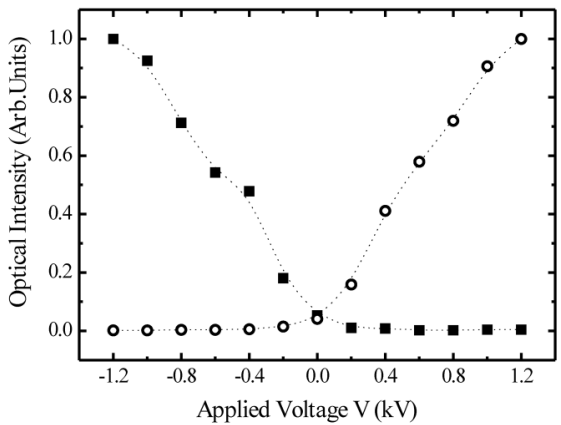

Applied Voltage V $(\mathrm{kV})$
Fig. 3. Switching through vertically stacked oppositely charged needles. (a) Input launch distribution; (b) output soliton formation; (c) the two output distributions for top, 1.2 and bottom, $-1.2 \mathrm{kV}$. Right, switching comparison (see Fig. 2).

at the output of the crystal in the positions that correspond to the two guiding patterns. The asymmetry in the switching behavior is a consequence of the asymmetry in the writing dynamics. The equivalent extinction ratio is $\eta>18 \mathrm{~dB}$.

A second practical switching method is to orient the oppositely charged pair along the $y$ axis, i.e., in a direction perpendicular to the biasing field. The two needles are generated as above, except that here the crystal shift $\Delta y \simeq 20 \mu \mathrm{m}$ is chosen to be the smallest possible that will permit a well-defined formation of the two needles. In fact, whereas long-range interaction mediated by lobes is absent, along the $y$ axis there are no intermediate structures that allow for screening of the antiguiding associated with the oppositely charged member of the pair. ${ }^{13,14}$ To avoid asymmetries that are not intrinsic to this configuration we made the system undergo a two-step dynamic soliton formation process: The system is allowed in each step to progress only halfway to the needle plateau; then it is shifted, the bias is inverted, and so forth. Finally, the crystal is aligned with the input launch read signal positioned between the two soliton patterns, and no further crystal repositioning occurs. Results are shown in Fig. 3. In particular, in Fig. 3(c) two 12- $\mu$ m-sized output modes are shown, one for $V=V_{\text {sol }}$ and the other for $V=-V_{\text {sol }}$. The signal beam is thus guided alternately in the positive and the negative channels. First we note that mode switching is achieved in a direction perpendicular to the biasing field: This is a natural consequence of electroholography that is not possible with other switching techniques, even on photorefractive time scales. Furthermore, the output mode distance is approximately $40 \mu \mathrm{m}$, larger than both for the previous $x$-oriented case and the distance $\Delta y \simeq 20 \mu \mathrm{m}$ between the two soliton-supporting patterns [Figs. 3(b) and 3(c)]. This effect is possibly ascribable to a moreelaborate mode coupling, given that the input read beam is not aligned with either needle pattern (to increase symmetry). Increasing the read level to $V>V_{\text {sol }}$ ameliorates the mode structure. Intensity switching measurements as a function of reading voltage $V$, analogous to those shown at the right in Fig. 2 and illustrated in Fig. 3, show greater symmetry and an extinction ratio $\eta>20 \mathrm{~dB}$.

In conclusion, we have reported experiments that demonstrate electro-optical switching based on photorefractive needles, opening the way to integrated, rewritable, fast beam manipulation in bulk samples.

This research was funded by the Italian Istituto Nazionale di Fisica della Materia through the Soliton Electro-Optic Structures project and in part by the Italian administration through the Cofunding 2001 and Athenaeum 2001 initiatives. A. J. Agranat was supported by the Ministry of Science of the State of Israel.

\section{References}

1. M. Segev, B. Crosignani, A. Yariv, and B. Fischer, Phys. Rev. Lett. 68, 923 (1992).

2. M. Segev, M. Shih, and G. C. Valley, J. Opt. Soc. Am. B 13, 706 (1996).

3. S. Trillo and W. Torruellas, eds., Spatial Solitons (Springer-Verlag, Berlin, 2002), Chaps. 4 and 5.

4. M. Shih, M. Segev, G. C. Valley, G. Salamo, B. Crosignani, and P. Di Porto, Electron. Lett. 31, 826 (1995).

5. E. DelRe, M. Tamburrini, M. Segev, E. Refaeli, and A. J. Agranat, Appl. Phy. Lett. 73, 16 (1998).

6. M. Morin, G. Duree, G. Salamo, and M. Segev, Opt. Lett. 20, 2066 (1995).

7. S. Lan, E. DelRe, Z. G. Chen, M. F. Shih, and M. Segev, Opt. Lett. 24, 475 (1999).

8. J. Petter and C. Denz, Opt. Commun. 188, 55 (2001).

9. S. Lan, M. F. Shih, G. Mizell, J. A. Giordmaine, Z. G. Chen, C. Anastassiou, J. Martin, and M. Segev, Opt. Lett. 24, 1145 (1999).

10. S. Lan, J. A. Giordmaine, M. Segev, and D. Rytz, Opt. Lett. 27, 737 (2002).

11. M. Balberg, M. Razvag, S. Vidrio, E. Refaeli, and A. J. Agranat, Opt. Lett. 21, 1544 (1996).

12. E. DelRe, M. Tamburrini, and A. J. Agranat, Opt. Lett. 25, 963 (2000).

13. E. DelRe, A. Ciattoni, and A. J. Agranat, Opt. Lett. 26, 908 (2001).

14. C. M. Gomez-Sarabia, P. A. Marquez-Aguilar, J. J. Sánchez-Mondragón, S. Stepanov, and V. Vysloukh, J. Opt. Soc. Am. B 13, 2767 (1996). 